\title{
Top Quark Production Cross Section Measurements *
}

\author{
L. Massa ${ }^{\mathrm{a}, \mathrm{b}}$, on behalf of the ATLAS collaboration \\ ${ }^{a}$ INFN Sezione di Roma Tor Vergata, Roma, Italy \\ ${ }^{b}$ Dipartimento di Fisica, Università di Roma Tor Vergata, Roma, Italy
}

\begin{abstract}
Measurements of the inclusive and differential cross-sections for top-quark pair and single top production cross sections in proton-proton collisions with the ATLAS detector at the Large Hadron Collider are presented at centre-ofmass energies of $8 \mathrm{TeV}$ and $13 \mathrm{TeV}$.

The inclusive measurements reach high precision and are compared to the best available theoretical calculations. These measurements, including results using boosted tops, probe our understanding of top-pair production in the TeV regime. The results are compared to Monte Carlo generators implementing LO and NLO matrix elements matched with parton showers and NLO QCD calculations.

For the t-channel single top measurement, the single top-quark and anti-top-quark total production cross-sections, their ratio, as well as differential cross sections are also presented. A measurement of the production cross section of a single top quark in association with a $W$ boson, the second largest single-top production mode, is also presented. Finally, measurements of the properties of the $W t b$ vertex allow to set limits on anomalous couplings. All measurements are compared to state-of-the-art theoretical calculations.
\end{abstract}

Keywords: top quark, cross section, $t \bar{t}$, single top

\section{The top quark}

The top quark, discovered in 1995 by both the CDF [1] and D0 [2] collaborations at the Tevatron collider, is the heaviest known particle of the Standard Model. Its mass of $m_{t}=173.3 \pm 0.7 \mathrm{GeV}$ is linked to a series of important properties.

First of all, the top quark is the only quark which decays before hadronizing, giving the unique possibility to study a "bare" quark.

In addition, the radiative corrections to the Higgs boson mass binds the masses of top quark, $W$ boson and Higgs boson, allowing important consistency tests on the Standard Model.

Finally, the top quark plays a special role in the electroweak symmetry breaking and in Beyond the Standard

\footnotetext{
*Talk given at 20th International Conference in Quantum Chromodynamics (QCD 17), 3 - 7 july 2017, Montpellier - FR

Email address: lorenzo.massa@cern.ch (L. Massa)
}

Model physics theories, thanks to its large Yukawa coupling with the Higgs boson. Indeed, many BSM theories involve new particles with large couplings to top quarks and $t \bar{t}$ resonances with the invariant mass in the $\mathrm{TeV}$ region, making studies on the $t \bar{t}$ differential cross section important to investigate possible anomalous effects.

In proton colliders like the Large Hadron Collider, the production of $t \bar{t}$ pairs is mainly due to gluon fusion (90\% at $\sqrt{s}=13 \mathrm{TeV}$ ) and quark-antiquark annihilation. Almost every top quark decays into a $W$ boson and a $b$ quark pair, and it is possible to have three different $t \bar{t}$ final states, depending on the decay of the $W \mathrm{~s}$ in a lepton-neutrino or a quark-antiquark pair: the hadronic channel, the lepton+jets channel and the di-lepton channel.

In this overview some of the most recent measurements of top pair and single top production cross section, obtained by the ATLAS Collaboration using data collected in 2012 (with an integrated luminosity of $L=$ 


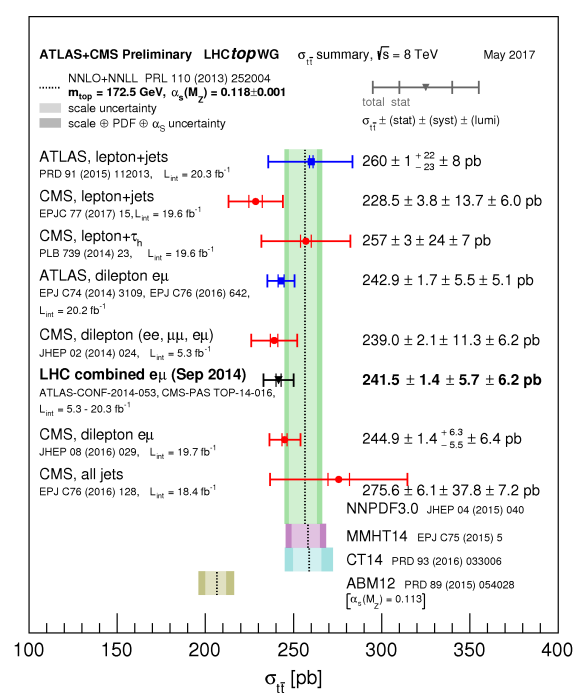

(a)

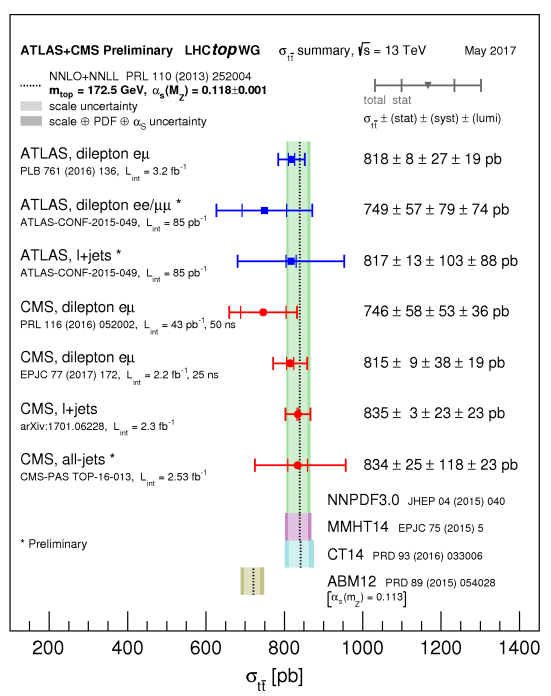

(b)

Figure 1: Summary of the $t \bar{t}$ inclusive cross section measurements performed by ATLAS and CMS at (a) $8 \mathrm{TeV}$ and (b) 13 TeV [4].

$20.3 \mathrm{fb}^{-1}$ at $\sqrt{s}=8 \mathrm{TeV}$ ) and in 2015 (with an integrated luminosity of $L=3.2 \mathrm{fb}^{-1}$ at $\sqrt{s}=13 \mathrm{TeV}$ ).

The description of the ATLAS detector and its performances can be found in [3].

\section{2. $t \bar{t}$ cross section}

The inclusive cross section has been measured by the ATLAS Collaboration in several ways, obtaining a good agreement with the Standard Model prediction, with experimental uncertainties smaller than the theoretical ones. A summary of all the measurements performed by ATLAS and CMS can be seen in figure 1 [4].

All these results have been obtained through cut based analysis, with different cuts depending on the studied final state.

\section{Lepton+jets channel}

Depending on the involved energies, there could be two different topologies, leading to different analysis strategies. In the case referred as resolved topology, the top quark has a momentum lower than $300 \mathrm{GeV}$, its decay products are well separated and can be reconstructed individually. This case the events are usually selected looking for one charged lepton, at least four jets (two of them compatible with the presence of $b$ quarks) and a certain amount of missing transverse energy, while the $t \bar{t}$ system is reconstructed through a kinematic fit.
For higher momentum top quarks, their decay products tend to become more collimated, making the previous reconstruction techniques less efficient. In this case, usually known as boosted topology, the events are selected looking for one charged lepton, at least one jet, a certain amount of missing transverse energy and the hadronically decaying top quark is reconstructed as a single large radius jet.

The main background of the lepton+jets final state is represented by $W+$ jets, QCD multijet and single top events. The branching ratio of this channel is $45 \%$.

\section{Dilepton (e $\mu)$ channel}

In this case events are selected looking for one electron and one muon of opposite charge, at least two jets (one compatible with the presence of a $b$ quark) and a large missing transverse energy. The $t \bar{t}$ is then reconstructed making use of kinematic assumptions.

The main background of the dilepton final state is represented by $Z / \gamma+$ jets, diboson and single top events. The branching ratio of this channel is $10 \%$.

\section{All hadronic channel}

In these case most of the studied events are in the boosted topology, so the events are selected looking for two large radius jets, at least two jets and at least two $b$-jets (one inside each large radius jet).

The main background of the all hadronic final state is represented by a large amount of QCD multijet events. 


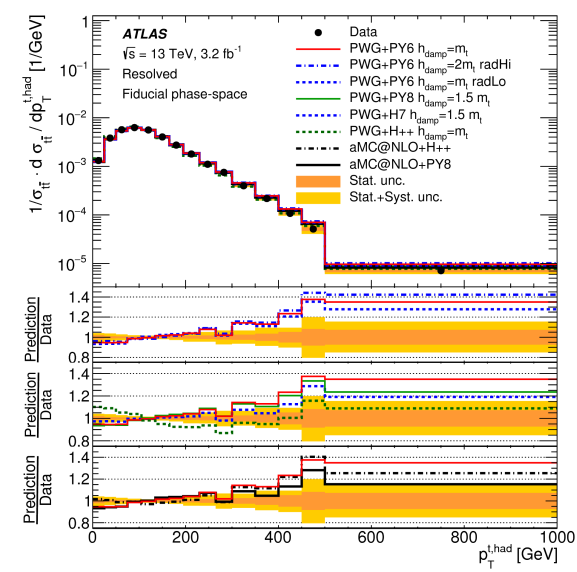

(a)

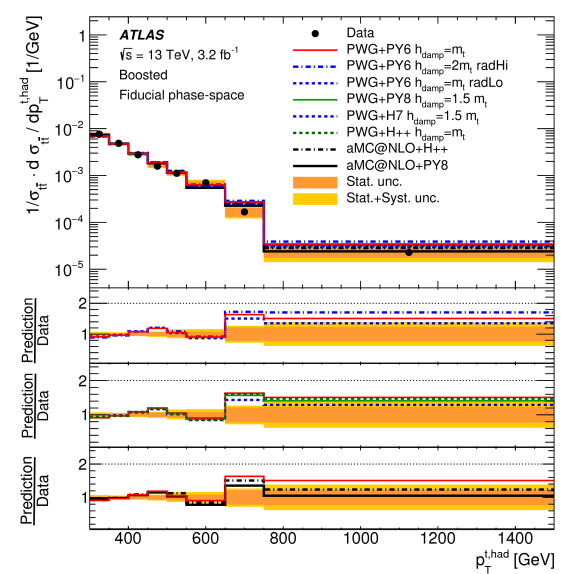

(b)

Figure 2: Fiducial phase-space relative differential cross-sections as a function of the transverse momentum of the hadronic top quark (a) in the resolved topology and (b) in the boosted topology with $\sqrt{s}=13 \mathrm{TeV}$, compared with the Standard Model predictions obtained with different Monte Carlo generators. The yellow bands indicate the total uncertainty of the data in each bin. The lower three panels show the ratio of the predictions to the data [11].

The branching ratio of this channel is $45 \%$.

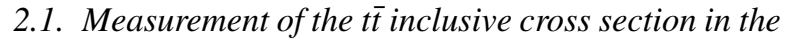 ex channel}

Among all the inclusive cross section measurements, the most precise is the one obtained in the final state with oppositely charged $e \mu$ pair.

This measurement has been performed using the data collected in 2011 with a center of mass energy of $7 \mathrm{TeV}$ and an integrated luminosity of $4.6 \mathrm{fb}^{-1}$, and the data collected in 2012 with a center of mass energy of $8 \mathrm{TeV}$ and $20.3 \mathrm{fb}^{-1}$ [5], as well as the data collected in 2015 with a center of mass energy of $13 \mathrm{TeV}$ and $3.2 \mathrm{fb}^{-1}[6]$. Counting the number of total events containing exactly one or two $b$-tagged jets, it has been possible to extract the inclusive cross section $\sigma_{t \bar{t}}$, as well as the $b$-tagging efficiency, with a significant reduction of the associated systematic uncertainties. The cross section is measured in the fiducial phase space, with one electron and one muon with $p_{T}>25 \mathrm{GeV}$ and $|\eta|<2.5$, and extrapolated to the total phase space, obtaining:

$$
\begin{array}{lr}
\sigma_{t \bar{t}}^{\text {total }}(7 \mathrm{TeV})= & 182.9 \pm 7.1 \mathrm{pb}, \\
\sigma_{t \bar{t}}^{\text {total }}(8 \mathrm{TeV})= & 242.9 \pm 8.8 \mathrm{pb}, \\
\sigma_{t \bar{t}}^{\text {total }}(13 \mathrm{TeV})= & 818 \pm 36 \mathrm{pb} .
\end{array}
$$

These results are consistent with what is expected by the theoretical QCD calculations at the NNLO[7]:

$$
\begin{array}{lr}
\sigma_{t \bar{t}}^{t h}(7 \mathrm{TeV})= & 177.3_{-10.8}^{+10.1} \mathrm{pb}, \\
\sigma_{t \bar{t}}^{t h}(8 \mathrm{TeV})= & 252.9_{-13.1}^{+13.3} \mathrm{pb}, \\
\sigma_{t \bar{t}}^{t h}(13 \mathrm{TeV})= & 832.9_{-46}^{+40} \mathrm{pb} .
\end{array}
$$

\section{2. $t \bar{t}$ differential cross section measurements}

The large integrated luminosity at the LHC allowed to perform precision differential cross section measurements, giving the possibility to search for unexpected discrepancies linked to physics Beyond Standard Model.

The differential $t \bar{t}$ cross section has been measured by the ATLAS detector at $8 \mathrm{TeV}$ [8-10] and $13 \mathrm{TeV}$ [11$13]$, as a function of the mass $\left(m_{t \bar{t}}\right)$, transverse momentum $\left(p_{T, t \bar{t}}\right)$ and absolute rapidity $\left(\left|y_{t t}\right|\right)$ of the system and the transverse momentum of the top quark $\left(p_{T, t}\right)$ with the resolved approach, and as a function of the transverse momentum $\left(p_{T, t}\right)$ and absolute rapidity $\left(\left|y_{t}\right|\right)$ of the top quark with the boosted approach. In all the cases, the measurement has been performed through a cutbased analysis in all the possible $t \bar{t}$ final states, extracting the reconstructed kinematic distributions and correcting them for detector effects using unfolding techniques.

The results have been compared to next-to-leadingorder (NLO) calculations from Monte Carlo generators, finding a good agreement with the Standard Model expectations. The only exception is represented by the data distribution for $p_{T, t}$, which appears to be softer than the NLO prediction, especially in the tail of high $p_{T}$ top quarks. This tendency has been found first at $8 \mathrm{TeV}$ in the lepton+jets channel, and then confirmed at $13 \mathrm{TeV}$ in all the possible final states. In figure 2 it is possible to see the normalized differential cross section obtained at $13 \mathrm{TeV}$ in the lepton+jets channel at particle level in a fiducial phase space. 


\section{Extrapolation at parton level}

In order to make comparison with other experiments and with the theoretical QCD calculations, the measurements can be extrapolated to the whole phase space at parton level. In figure 3 it is possible to see the extrapolation obtained for the differential $t \bar{t}$ cross section with respect to the transverse momentum of the single top quark at $8 \mathrm{TeV}$ [4]. As can be seen, there is a good agreement between the results obtained by ATLAS and CMS, and the agreement with the theoretical prediction is better than the particle level case.

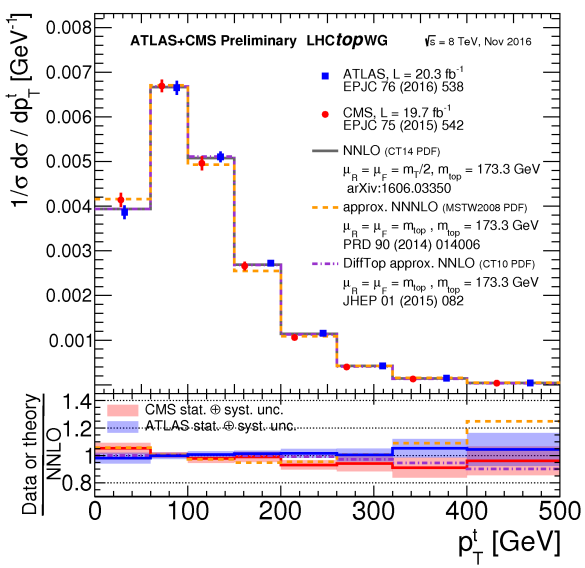

Figure 3: Full phase-space normalised differential ttbar cross-section as a function of the transverse momentum of the top quark. The CMS and ATLAS results are compared to the NLO and NNLO calculations[14] [4].

\section{Single top cross section measurement}

The ATLAS experiment measured several inclusive single top production cross section, at $\sqrt{s}=7,8$ and $13 \mathrm{TeV}$, in all the three different production channels ( $t$-channel, $W t$ production and $s$-channel), obtaining a good agreement with the Standard Model predictions. A summary of all the measurements performed by ATLAS and CMS can be seen in figure 4 [4].

The measurement of the $W t$ production and the $t$ channel cross section will be shown in detail in the following sections.

\subsection{Wt production total cross section}

The $W t$ production total cross section has been measured at $8 \mathrm{TeV}$ [15] and $13 \mathrm{TeV}$ [16] of center-of-mass energy, in the dilepton channel where

$$
t W^{-} \rightarrow\left(W^{+} b\right) W^{-} \rightarrow\left(l^{+} v b\right) l^{-} \bar{v} .
$$

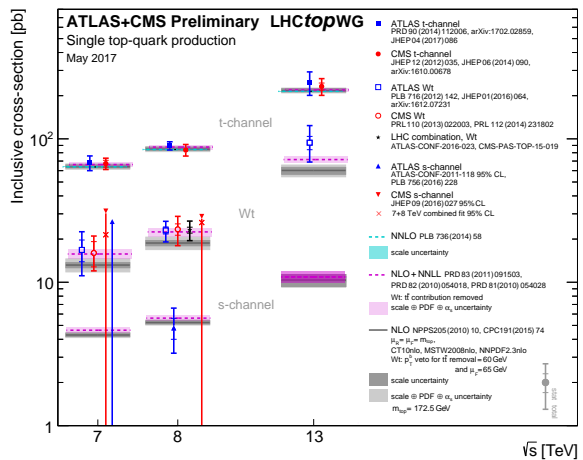

Figure 4: Summary of ATLAS and CMS measurements of the single top production cross-sections in various channels as a function of the center of mass energy. The measurements are compared to theoretical calculations based on: NLO QCD, NLO QCD complemented with NNLL resummation and NNLO QCD ( $t$-channel only) [4].

The measurement is performed through a Boosted Decision Tree, in order to separate the $W t$ signal from the $t \bar{t}$ background obtaining discriminant variables which are used in a profile likelihood fit to calculate the $W t$ cross section. The results obtained are:

$$
\begin{array}{lr}
\sigma_{W t}(8 \mathrm{TeV})= & 23.0_{-3.8}^{+3.6} \mathrm{pb} \\
\sigma_{W t}(13 \mathrm{TeV})= & 94_{-24}^{+30} \mathrm{pb}
\end{array}
$$

The results are compatible with the standard model expectations, which are:

$$
\begin{array}{lr}
\sigma_{W t}^{t h}(8 \mathrm{TeV})= & 22.37 \pm 1.52 \mathrm{pb}, \\
\sigma_{W t}^{t h}(13 \mathrm{TeV})= & 71.7 \pm 3.8 \mathrm{pb}
\end{array}
$$

\section{2. $t$-channel total and differential cross section}

Also the $t$-channel single top quark production cross section has been measured in the lepton+jets topology, making use of a maximum likelihood fit of a multivariate Neural Network discriminant trained with the most sensitive variables.

The measurement has been performed at $8 \mathrm{TeV}$ [17] and $13 \mathrm{TeV}$ [18], obtaining the production cross section for top quarks, antitop quarks and the ratio between the two cross sections.

The results obtained with the $8 \mathrm{TeV}$ sample of 20.3 $\mathrm{fb}^{-1}$ are

$$
\begin{aligned}
\sigma_{t q}(8 \mathrm{TeV}) & =57.7_{-3.8}^{+4.3} \mathrm{pb}, \\
\sigma_{\bar{t} q}(8 \mathrm{TeV}) & =32.9_{-2.7}^{+3.0} \mathrm{pb}, \\
R_{t}=\frac{\sigma_{t q}}{\sigma_{\bar{t} q}}(8 \mathrm{TeV}) & =1.72 \pm 0.09,
\end{aligned}
$$


while the results obtained with the $13 \mathrm{TeV}$ sample of 3.2 $\mathrm{fb}^{-1}$ are

$$
\begin{array}{rr}
\sigma_{t q}(13 \mathrm{TeV}) & =156 \pm 28 \mathrm{pb}, \\
\sigma_{\bar{q} q}(13 \mathrm{TeV}) & =91 \pm 19 \mathrm{pb}, \\
R_{t}=\frac{\sigma_{t q}}{\sigma_{\overline{t q}}}(13 \mathrm{TeV}) & =1.72 \pm 0.20 .
\end{array}
$$

All the results are in good agreement with the Standard Model predictions.

In the case of the $8 \mathrm{TeV}$ sample, the high statistics allowed a measurement of a differential top and antitop production cross section, with respect to the transverse momentum and rapidity of the single top, using a maximum likelihood fit and an unfolding procedure at parton and particle level and obtaining results in agreement with the Standard Model.

\subsection{Wtb vertex structure setting limits on anomalous couplings}

The $t$-channel can be used also to measure the polarity of the top quarks. Indeed, according to the Standard Model, top quarks should be highly polarized

$$
P(t)=0.91 ; P(\bar{t})=-0.86
$$

The polarity is linked to the angular asymmetries, which can be observed in the $t$-channel.

$$
A_{F B}=\frac{N_{\uparrow}-N_{\downarrow}}{N_{\uparrow}+N_{\downarrow}}=\frac{N(\cos \theta>0)-N(\cos \theta<0)}{N(\cos \theta>0)+N(\cos \theta<0)}
$$

ATLAS performed such measurement using a data sample with a center-of-mass energy of $8 \mathrm{TeV}$ and an integrated luminosity of $20.3 \mathrm{fb}^{-1}$ [19], selecting $t$ channel events with one isolated charged lepton, large missing transverse energy and two jets (one tagged as a $b$-jet).

The angular asymmetries are measured with respect to the top quark and the $\mathrm{W}$ boson's spin quantisation axes, following the coordinate scheme shown in figure 5.

The measured angular asymmetries are corrected through a parton level unfolding and show a good agreement with the Standard Model predictions, as well as the resulting top-quark and $W$ boson's polarisations.

In particular, the most interesting result comes from the possibility to set limits on anomalous couplings using the asymmetries $A_{F B}^{N}$ and $A_{F B}^{l}$, whose angular observables are the cosines of the angle $\theta_{l}^{N}$ (between the top quark spin axis and the normal direction of the charged lepton) and the angle $\theta_{l}$ (between the top quark spin axis and the direction of the charged lepton).

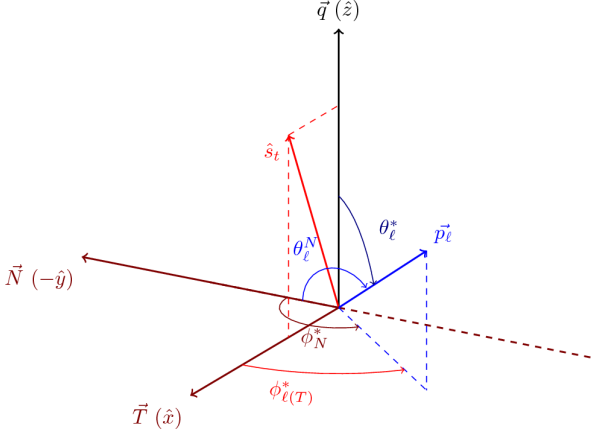

Figure 5: Coordinate system and angles used to define the $W$ boson spin observables and their related angular asymmetries in the decay of polarised top quarks. The $W$ boson momentum $q$ in the top quark rest frame defines the $z$-axis; the top quark spin direction $s_{t}$, taken along the spectator quark momentum in the top quark rest frame, defines the $x z$ plane [19].

Indeed, the $W t b$ interaction can be generalized in this way:

$$
\begin{aligned}
L_{W t b}= & -\frac{g}{\sqrt{2}} \bar{b} \gamma^{\mu}\left(V_{L} P_{L}+V_{R} P_{R}\right) t W_{\mu}^{-} \\
& -\frac{g}{\sqrt{2}} \bar{b} \frac{i \sigma^{\mu v} q_{v}}{m_{W}}\left(q_{L} P_{L}+q_{R} P_{R}\right) t W_{\mu}^{-} \\
& + \text {h.c. }
\end{aligned}
$$

In the Standard Model, the only component which is present is $V_{L}$, which is the $V_{t b}$ term of the CKM matrix. However, a complex $g_{R}$ could imply anomalous and CPviolating couplings.

$A_{F B}^{N}$ and $A_{F B}^{l}$ are the most sensitive observables to variations of $\operatorname{Im}\left[g_{R}\right]$ and so they are useful to probe the presence of anomalous couplings. Without making any assumption on $\operatorname{Im}\left[g_{R}\right]$, the values obtained are

$$
\begin{aligned}
& A_{F B}^{N}=-0.04 \pm 0.04 \text { and } \\
& A_{F B}^{l}=0.49 \pm 0.06,
\end{aligned}
$$

which, assuming $V_{L}=1$ and $V_{R}=g_{L}=\operatorname{Re}\left[g_{R}\right]=0$ lead to the following limits on the imaginary part of $g_{R}$ :

$$
\operatorname{Im}\left[g_{R}\right] \in[-0.18,0.06]
$$

at the $95 \%$ confidence level.

\section{Summary}

ATLAS has performed several $t \bar{t}$ and single top quark cross section measurements to make stringent tests on Standard Model, both at $\sqrt{s}=8 \mathrm{TeV}$ and $13 \mathrm{TeV}$. Especially for the events at $13 \mathrm{TeV}$, dedicated studies on boosted topologies allowed a better description of many events. 
Most of the measurements are limited by systematic uncertainties and all the results agree with the Standard Model, with the only exception of the $t \bar{t}$ differential cross section with respect to $p_{T, t}$, where the data distribution is slightly softer then the theoretical prediction, especially for top quarks with a $p_{T}$ greater than $200 \mathrm{GeV}$.

With the new runs, the precision of these results is expected to be refined as an effect of the higher statistics.

Any additional information, together with the updated list of all the ATLAS results on top physics, is available at the ATLAS top public result webpage [20].

\section{References}

[1] CDF Collaboration, Phys. Rev. Lett., 74 (1995) 2626.

[2] D0 Collaboration), Phys. Rev. Lett., 74 (1995) 2632.

[3] ATLAS Collaboration, JINST, 3 (2008) S08003.

[4] ATLAS Collaboration, Summary plots from the ATLAS Top physics group, https://atlas.web.cern.ch/Atlas/ GROUPS/PHYSICS/CombinedSummaryPlots/TOP/.

[5] ATLAS Collaboration, Eur. Phys. J. C 76 (2016) 642.

[6] ATLAS Collaboration, Phys. Lett. B761 (2016) 136.

[7] Czakon M. and Mitov A., Comput. Phys. Commun., 185 (2014) 2930.

[8] ATLAS Collaboration, Eur. Phys. J. C76 (2016) 538.

[9] ATLAS Collaboration, Phys. Rev. D93 (2016) 032009.

[10] ATLAS Collaboration, Phys. Rev. D94 (2016) 092003.

[11] ATLAS Collaboration, arXiv:1708.00727 [hep-ex] (submitted to JHEP).

[12] ATLAS Collaboration, Eur. Phys. J. C77 (2017) 299.

[13] ATLAS Collaboration, ATLAS-CONF-2016-100, https://cds.cern.ch/record/2217231.

[14] Michal Czakon, David Heymes, Alexander Mitov, JHEP04(2017)071.

[15] ATLAS Collaboration, JHEP 01 (2016) 064.

[16] ATLAS Collaboration, arXiv: 1612.07231 [hep-ex] (submitted to JHEP)

[17] ATLAS Collaboration, Eur. Phys. J. C 77 (2017) 531.

[18] ATLAS Collaboration, JHEP04(2017)086.

[19] ATLAS Collaboration, JHEP04 (2017) 124.

[20] ATLAS Collaboration, Top Public Results, https://twiki.cern.ch/twiki/bin/view/AtlasPublic/TopPublicResults. 